\title{
Uncovering flux line correlations in superconductors by reverse monte carlo refinement of neutron scattering data
}

\author{
Laver, M.; Forgan, E.M.; Abrahamsen, Asger Bech; Bowell, C.; Geue, T.; Cubitt, R.
}

Published in:

Physical Review Letters

Link to article, DOI:

10.1103/PhysRevLett.100.107001

Publication date:

2008

Document Version

Publisher's PDF, also known as Version of record

Link back to DTU Orbit

Citation (APA):

Laver, M., Forgan, E. M., Abrahamsen, A. B., Bowell, C., Geue, T., \& Cubitt, R. (2008). Uncovering flux line correlations in superconductors by reverse monte carlo refinement of neutron scattering data. Physical Review Letters, 100(10), 107001. https://doi.org/10.1103/PhysRevLett.100.107001

\section{General rights}

Copyright and moral rights for the publications made accessible in the public portal are retained by the authors and/or other copyright owners and it is a condition of accessing publications that users recognise and abide by the legal requirements associated with these rights.

- Users may download and print one copy of any publication from the public portal for the purpose of private study or research.

- You may not further distribute the material or use it for any profit-making activity or commercial gain

- You may freely distribute the URL identifying the publication in the public portal 


\title{
Uncovering Flux Line Correlations in Superconductors by Reverse Monte Carlo Refinement of Neutron Scattering Data
}

\author{
M. Laver, ${ }^{1,2,3,4}$ E. M. Forgan, ${ }^{2}$ A. B. Abrahamsen, ${ }^{5}$ C. Bowell, ${ }^{2}$ Th. Geue, ${ }^{6}$ and R. Cubitt ${ }^{3}$ \\ ${ }^{1}$ NIST Center for Neutron Research, Gaithersburg, Maryland 20899, USA \\ ${ }^{2}$ School of Physics and Astronomy, The University of Birmingham, Birmingham B15 2TT, United Kingdom \\ ${ }^{3}$ Institut Laue-Langevin, BP 156, F-38042 Grenoble, France \\ ${ }^{4}$ University of Maryland, College Park, Maryland 20742, USA \\ ${ }^{5}$ Ris $\phi$ National Laboratory, DTU, Frederiksborgvej 399, DK-4000 Roskilde, Denmark \\ ${ }^{6}$ Paul Scherrer Institut, Villigen PSI, CH 5232, Switzerland
}

(Received 2 November 2007; published 12 March 2008; corrected 31 March 2008)

\begin{abstract}
We describe the use of reverse Monte Carlo refinement to extract structural information from angleresolved data of a Bragg peak. Starting with small-angle neutron scattering data, the positional order of an ensemble of flux lines in superconducting $\mathrm{Nb}$ is revealed. We discuss the uncovered correlation functions in the light of topical theories, in particular, the "Bragg glass" paradigm.
\end{abstract}

PACS numbers: 74.25.Qt, 05.10.Ln, 61.05.F-, 61.43.-j

While scattering data usually contain detailed structural information about a system, the direct extraction of correlation functions and microscopic quantities from the data is often difficult. This has led to the development of computational methods such as reverse Monte Carlo (RMC) refinement [1] that work by going from possible structures to the data while enforcing obvious physical conditions. This is exemplified by the derivation of magnetic moment orientations from total neutron scattering data [2]. Up to now, RMC refinement has been applied predominantly with measurements as a function of the magnitude of the scattering vector $\mathbf{k}$ from powder samples [2], amorphous alloys [3], or liquids [4]. Here we extend the RMC approach to provide structural insight into weakly disordered crystalline samples, using angle-resolved scattering data obtained by rotating the sample through a Bragg peak. We examine the Abrikosov flux line (FL) lattice of a Type-II superconductor [5], accurately reproducing small-angle neutron scattering (SANS) observations. Our extended technique is readily applicable to other two-dimensional (2D) systems.

Our motivation is to test competing theories on the influence of disorder upon the FL arrangement. Long ago, Larkin showed that any disorder-as induced by pinning of FL's to randomly distributed defects or impurities in the underlying crystal-would destroy long-range order due to a linear increase of elastic distortions with FL separation [6]. More recently it was realized that for weak quenched disorder, a purely Gaussian model with random forces becomes inadequate beyond the Larkin length $R_{\xi}$ where displacements become comparable to the characteristic length scale $\xi$ of the pinning potential [7-9]. Beyond the Larkin regime the FL's are anticipated to begin to behave collectively as an elastic manifold in a "random manifold" (RM) regime [7]. At asymptotic scales larger than the crossover length $R_{A}$, in the absence of dislocations, the FL lattice periodicity [8] may result in a much slower, logarithmic increase of elastic distortions with distance giving Bragg peaks and algebraically decaying translational order-the so-called Bragg glass (BrG) phase [9]. Under strong disorder or high magnetic fields the $\mathrm{BrG}$ is destroyed by dislocations [10]. The properties of the ensuing vortex glass phases have yet to be resolved by experiment, though several isotropic, hexatic, and "fractured" phases have been conjectured [11-13]. A previous SANS study [14] has claimed to demonstrate the existence of a $\mathrm{BrG}$ phase from an analysis of Bragg peak intensity with the aid of a theoretical form factor. In contrast, we use a high-resolution SANS geometry particularly sensitive to in-plane FL correlations; our RMC approach then uncovers structural information contained in the peak shape without requiring an a priori model.

The principle of operation of RMC refinement, detailed elsewhere [1], is that positions of scattering centers in an ensemble are modified using a Monte Carlo procedure whereby a random modification is always accepted if the least-squares goodness-of-fit $\chi^{2}$ is reduced, or otherwise with a probability $\exp \left(-\Delta \chi^{2} / p\right)$. Traditionally the weighting $p=2$ [1]. The calculated scattered intensity $I_{\text {calc }}(\mathbf{k})$ is the convolution of the instrument resolution $Y(\mathbf{k})$ and the elastic structure factor $S(\mathbf{k})$

$$
I_{\text {calc }}=F Y \star S \quad \text { with } \quad S(\mathbf{k})=\left|\sum_{j}^{N} \mathrm{e}^{-\mathrm{i} \mathbf{k} \cdot \mathbf{r}_{j}}\right|^{2},
$$

where the constant of proportionality $F$ contains the form factor of an individual scatterer and $\mathbf{r}_{j}$ is the position of the $j$ th scatterer averaged over dynamical disorder. We take $F$ as independent of $|\mathbf{k}|$ over the small range of $|\mathbf{k}|$ considered here.

In previous RMC implementations [1-4] a Bragg reflection associated with reciprocal lattice vector $\mathbf{G}$ has been characterized via $\mathbf{k}^{\prime} \| \mathbf{G}$ where $\mathbf{k}=\mathbf{G}+\mathbf{k}^{\prime}$. Here we consider $\mathbf{k}^{\prime}$ tangential to $\mathbf{G}$ and examine the shape of the 
rocking curve measured as the sample is rotated by the rocking angle $\varphi$ through the Bragg peak at $\varphi_{0}$ (Fig. 1). We show how our RMC refinement generates a rocking curve $I_{\text {calc }}(\varphi)$ from $S(\mathbf{k})$ in Fig. 2(a).

Our FL measurements were performed on the SANS-II instrument at the Paul Scherrer Institut using neutrons of mean wavelength $\lambda=10.6 \AA$ and spread $\Delta \lambda / \lambda \simeq 0.06$, collimated over a distance of $6 \mathrm{~m}$ from our sample. The latter was an unannealed niobium single-crystal disc of diameter $12 \mathrm{~mm}$, thickness $4 \mathrm{~mm}$, with the disc and crystal [111] axes coincident, mounted inside a fixed cryomagnet providing a field of $200 \mathrm{mT}$ along the disc axis and perpendicular to the neutron beam. A motorized sample stick with a cryogenic gearbox was used to rotate the sample by $\varphi$ about the disc axis. To generate the rocking curve $I_{\text {exp }}(\varphi)$, neutrons diffracted at twice the Bragg $\theta$ in the lower half of the vertical scattering plane were integrated over an area roughly equal to the incident beam spot size on the $2 \mathrm{D}$ detector placed $6 \mathrm{~m}$ from the sample. The measured Bragg spot size of $0.07^{\circ}$ is comparable with the angular spread $\nu \simeq 0.06^{\circ}$ of the incident beam, and considering $\Delta \lambda / \lambda$, this indicates poor resolution $\Delta k^{\prime} / G \approx$ 0.1 for $\mathbf{k}^{\prime} \| \mathbf{G}$. For $\mathbf{k}^{\prime}$ tangential to $\mathbf{G}$, our setup with field perpendicular to beam yields a much better resolution $Y(\varphi)$ that is effectively Gaussian of width $\nu$ (Fig. 1), corresponding to $\Delta k^{\prime} / G \simeq 0.001$. The FL ensemble was formed by applying the field above $T_{c} \simeq 9.2 \mathrm{~K}$ and cooling to $2.6 \mathrm{~K}$; weak disorder results from the small concentration of residual impurities in the unannealed $\mathrm{Nb}$ crystal, as reflected by an upper critical field $B_{c 2}(0) \approx 460 \mathrm{mT}$ of our sample slightly bigger than $B_{c 2}(0)=430 \mathrm{mT}$ typical of a pure sample [15].

As the FL's in $\mathrm{Nb}$ are effectively nearly straight rods of macroscopic length, we contract the longitudinal dimension of $\mathbf{r}_{j} \rightarrow \overline{\mathbf{r}}_{j}^{\perp}$ in (1), so $\overline{\mathbf{r}}_{j}^{\perp}$ represents the average position of the $j$ th FL in the plane perpendicular to the field

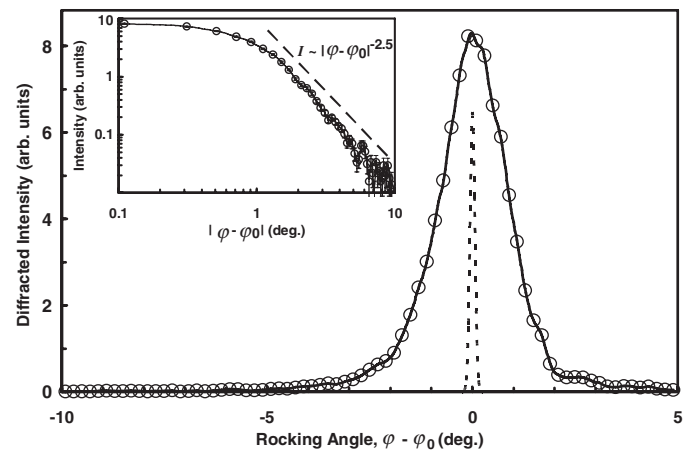

FIG. 1. Angular dependence of the diffracted neutron intensity from the flux line ensemble. The circles depict the experimental rocking curve $I_{\exp }(\varphi)$, while the solid line indicates $I_{\text {calc }}(\varphi)$ calculated from a RMC ensemble of $N=40015$ flux lines, after smoothing over the instrument resolution $Y(\varphi)$ illustrated by the thin dashed line. The inset replots these data on a double logarithmic scale; the thick dashed line is a guide to the eye showing an algebraic decay. direction. This averaging is valid if $\mathbf{r}_{j}^{\perp}$ varies sinusoidally or as a (Gaussian) random variable along the FL length, over the scale of the neutron coherence length [16] $\approx$ $10 \mu \mathrm{m} \gg$ FL lattice constant $a_{0}=109 \mathrm{~nm}$ at $200 \mathrm{mT}$. A static "Debye-Waller" factor $F_{\text {DW }}^{2}(|\mathbf{k}|) \equiv \mathrm{e}^{-\mathbf{k}^{2}\left\langle\mathbf{r}^{\perp 2}\right\rangle}$ is thus introduced into (1) and is absorbed by the factor $F$.

In Fig. 1 we illustrate the excellent agreement of the RMC generated neutron intensity $I_{\text {calc }}(\varphi)$ with the experimental data $I_{\exp }(\varphi)$ after a total of $980 N$ accepted moves. Each generated move is in a random direction and amplitude $\leq 0.01 a_{0}$. The RMC ensemble has size $N=40015$ FL's for all results depicted in the figures and is initiated as a near-perfect hexagonal FL lattice at the observed flux density $b=200 \mathrm{mT}$ constructed by perturbing an ideal lattice by a few (10 per FL) all-accepted moves. $N \gg$ 2000 is required to ensure that the intrinsic angular peak width $\simeq 1 / \sqrt{N}$ of the finite RMC configuration is less than that of our neutron data. We find the usual choice of $p=2$ [1] for the weighting of accepting a $\chi^{2}$-increasing move leads to quickly decaying positional order because almost every generated move is accepted. We use $p \simeq 0.03$, limiting the fraction of proposed $\chi^{2}$-increasing moves that are accepted to $\approx 50 \%$. We achieve similar results - as indicated by similar correlation functions calculated from the RMC ensembles - for reasonable variation of all computational parameters.

No knowledge of the form factor is needed if the value of $F$ in (1) is chosen to minimize $\chi^{2}$ throughout the RMC refinement. However, the order then fades slowly (Fig. 3) even after $\chi^{2}$ has been minimized, becoming unperceivable after $\approx 10^{5} \mathrm{~N}$ moves. Always working to maximize entropy, the RMC refinement is able through freedom in $F$ to shift scattering out of the first-order Bragg peak into the large fraction of remaining reciprocal space not encompassed by experimental data [Fig. 2(a)]. Even then, every RMC ensemble generated reproduces accurately the shape of the experimental rocking curve and from Fig. 3 we see they all yield the same form of the correlation functions. The slow decay of order with RMC run time can be restrained by applying local physical conditions on FL positions, such as a planarity constraint forbidding formation of dislocations by confining each FL to a cage defined by its six nearest neighbors [17]. We do not impose this constraint since only 35 of the 40015 FL's fall outside their nearest-neighbor cages after $610 \mathrm{~N}$ moves, the fraction increasing to $0.7 \%$ after $1740 \mathrm{~N}$ moves. This strongly implies our RMC results are consistent with a weakly disordered FL lattice free of dislocations.

The diminution of positional order is halted by bounding the scale factor $F$ in (1) to lie below a certain maximum value $m$. For our results in Figs. 1 and 2 and those denoted by " $F$ bounded" in Fig. 3, $F$ is limited below a value $m$ that corresponds to a form factor $h_{10}=70 \mathrm{mT}$ for the (10) FL Bragg reflection, estimated using the expected integrated intensity from 40015 perfectly arranged FL's in (1). Even though the static Debye-Waller factor $F_{\mathrm{DW}}$ was 

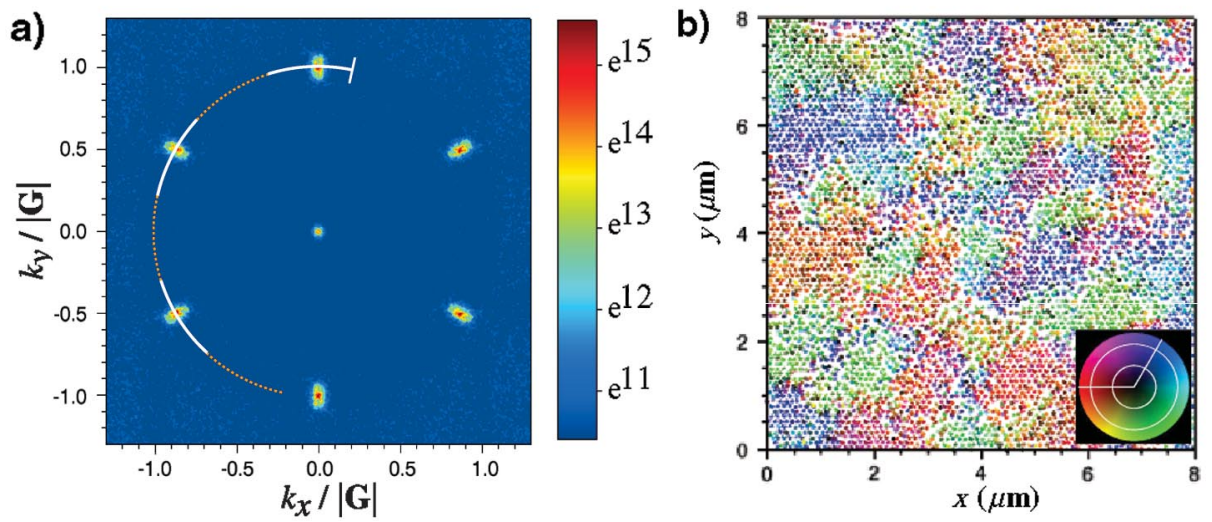

FIG. 2 (color). 2D (in-plane) maps from a RMC ensemble of $N=40015$ flux lines after $980 N$ moves. In (a) the structure factor $S(\mathbf{k})$ defined by (1) is plotted on a logarithmic color scale. The solid white arcs depict the reciprocal space encompassed by our experimental rocking curve (Fig. 1). The hexagonal FL lattice symmetry is preserved by duplicating our data at three angles $60^{\circ}$ apart. Between the first-order Bragg peaks zero scattered intensity was observed within experimental error. Thus, in these regions (marked by dotted orange arcs), the intensity to be fitted was set to zero with $\chi^{2}$ determined by the error. In calculating the neutron intensity $I_{\text {calc }}$, $S(\mathbf{k})$ is first integrated radially as shown by the white radial line, to account for the poor instrument resolution in this direction, before convolution with the much better tangential resolution $Y(\varphi)$. In (b) we show the displaced FL positions in a typical portion of the RMC ensemble. The color of each FL denotes the magnitude (lightness) and direction (hue) of its in-plane displacement $\mathbf{u}(\mathbf{R})$ from the corresponding perfect lattice, as described by the color wheel (inset) where white circles mark displacements of $0.2 a_{0}$ and $0.4 a_{0}$, and white radial lines depict the lattice basis vectors.

ignored for this estimate, it is consistent with $h_{10} / b \approx$ $10^{-1}$ found in early $\mathrm{Nb}$ neutron experiments [18].

In Fig. 3 we show the derived displacement correlator $B(R)=\left\langle\left\{\mathbf{u}_{j}-\mathbf{u}_{l}\right\}^{2}\right\rangle, \quad$ translational order $c_{G}(R)=$ $\left\langle\exp \left(\mathbf{i G} \cdot\left\{\mathbf{u}_{j}-\mathbf{u}_{l}\right\}\right)\right\rangle$ and orientational order $g_{6}(R)=$ $\left\langle\exp \left(\mathrm{i} 6\left\{\Upsilon_{j}-\Upsilon_{l}\right\}\right)\right\rangle$ where $\mathbf{u}_{j} \equiv \overline{\mathbf{r}}_{j}^{\perp}-\mathbf{R}_{j}$ is the displacement of the $j$ th FL; $R=\left|\mathbf{R}_{j}-\mathbf{R}_{l}\right|$ is the distance between $j$ th and $l$ th FL's of the corresponding perfect FL lattice; $\Upsilon_{j}$ is a nearest-neighbor bond angle relative to a fixed axis. $\langle\ldots\rangle$ denotes the average over the ensemble and over all six first-order reciprocal lattice vectors $\mathbf{G}$ or nearest-neighbor bonds where appropriate. At least two regimes are evident from the $B(R)$ [Fig. 3(a)] and $c_{G}(R)$ [Fig. 3(b)]. To date, the scale dependence of correlation functions has only been explored by elastic theory [7,9,19]. Accordingly we fit two-piece functions that are appropriate for describing RM and BrG behavior: $B(R)=\alpha_{0} R^{2 \zeta_{B}} h_{-}(R)+\left\{\alpha_{1} \ln R+\right.$ $\left.\alpha_{2}\right\} h_{+}(R) \quad$ and $\quad c_{G}(R)=\beta_{0} \exp \left(-R^{2} \zeta_{c} / \xi_{c}\right) h_{-}(R)+$ $\beta_{1} R^{-} \eta_{G} h_{+}(R) ; h_{ \pm}(R)=\frac{1}{2} \pm \frac{1}{2} \tanh \left(\left\{R-R_{A}\right\} / a_{0}\right)$ approximates a Heaviside step at the crossover $R_{A}$. These forms are fitted over $0<R<80 a_{0}$; the correlation function calculation is affected at large $R$ by the edges of the RMC ensemble that has radius $105 a_{0}$.

The correlation functions shown in Fig. 3 are described by run-invariant fit parameters $R_{A} \approx 15 a_{0}, \zeta_{c} \simeq 0.6, \xi_{c} \simeq$ $10 a_{0}, \zeta_{B}=0.2$, and $\eta_{G}=0.9$. We fit the orientational order by an algebraic form $g_{6}(R) \sim R^{-} \eta_{6}$. The persistence of long-range orientational order $\left(\eta_{6}=0.07 \ll \eta_{G}\right)$ while $c_{G}(R)$ falls more rapidly implies extended correlations in nearest-neighbor orientation, characteristic of the hexatic phases [12] observed here. At small scales $R<R_{A}$ our data strongly imply a RM regime, where FL's are collectively pinned but do not compete with each other for the same disorder energy minima. $c_{G}(R)$ is seen to decay exponentially [Fig. 3(b)] and displacements increase with roughness $\zeta_{B}=0.2$ [Fig. 3(a)], in excellent agreement with predicted RM regime values of 1/6 [20], 1/5 [7,21], and 0.17 [19]. Noting that crossover from Larkin to RM occurs when $B\left(R_{\xi}\right) \simeq \xi_{0}^{2}$, then with $\xi_{0} \approx 390 \AA$ in $\mathrm{Nb}$ we expect $R_{\xi} \approx 3 a_{0}$. At these small scales, the poor agreement seen in Fig. 3 to our fits describing larger scale regimes can thus be accounted for by a Larkin regime on the edge of our measurable limit.

At large scales $R>R_{A}$, the observed algebraic decay of the Bragg peak tails with exponent $\eta_{S} \simeq 2.5$ (Fig. 1), and of $c_{G}(R)$ [Fig. 3(b)] with exponent $\eta_{G}=0.9$ are in line with the approximation $\eta_{S} \approx 3-\eta_{G}$ expected for scattering vectors $\mathbf{k}$ in the vicinity of $\mathbf{G}$ where the structure factor is roughly the Fourier transform of $c_{G}(R)$ [19]. These algebraic decays and the slow logarithmic growth of displacements $B(R)$ [Fig. 3(a)] are consistent with a quasiordered $\mathrm{BrG}$ phase. However, our $\eta_{G}=0.9$ only approximates the predicted values of 1 and $\pi^{2} / 9$ [9], and 1.14 [19]. We note the region around $R_{A}$ is ill described by our two-piece fits [Fig. 3(a)]; details of the RM-BrG crossover regime have yet to be explored by theory. Our value of $B\left(R_{A}\right) \approx 0.2 a_{0}^{2}$ [Fig. 3(a)] also contrasts with the predicted $B\left(R_{A}\right) \simeq a_{0}^{2}$. We point out that in $\mathrm{Nb}$ the FL cores are larger compared to $a_{0}$ than in other superconductors, so the crossover $R_{A}$ where FL's start competing for the same disorder energy minima may occur at smaller scales.

From Fig. 2(b) it is evident that FL's group together in regions of similar displacements, with large relative displacements between these regions. Such "fracturing" is also seen in large-scale decoration images [22]. In this 


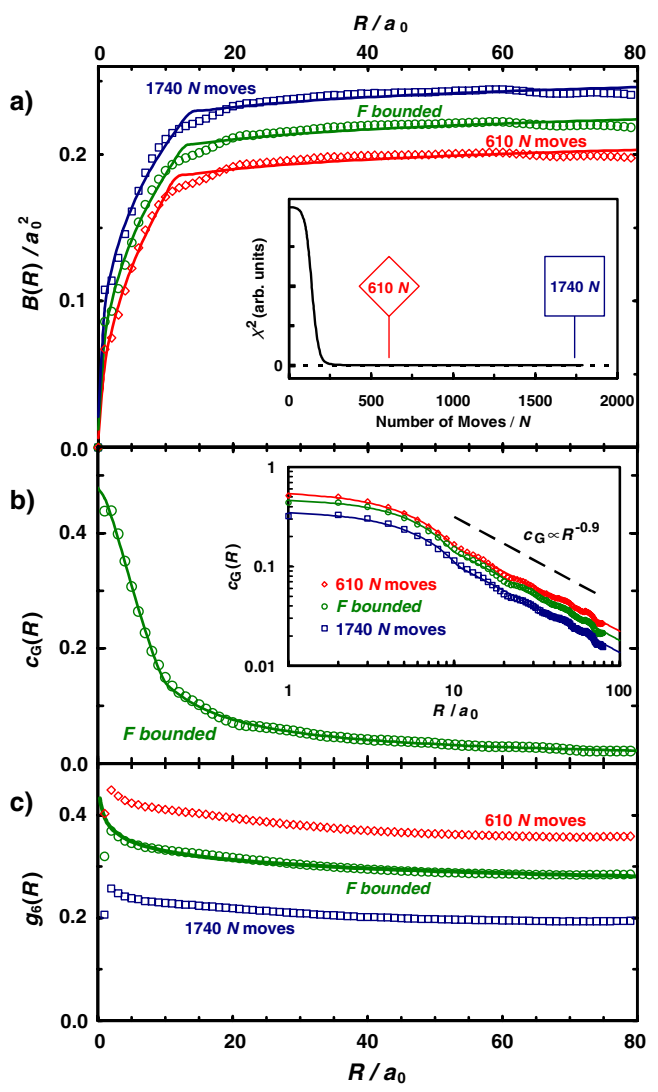

FIG. 3 (color online). Correlation functions derived from RMC ensembles of $N=40015$ FL's with the scale factor $F$ in (1) chosen to minimize $\chi^{2}$ after $610 N$ moves (red diamonds) and $1740 N$ moves (blue squares), and with $F$ bounded as described in the text after $980 \mathrm{~N}$ moves (green circles). The displacement correlator $B(R)$ is shown in (a); the inset shows the goodness-offit statistic $\chi^{2}$ describing how well the calculated intensity $I_{\text {calc }}(\varphi)$ reproduces the experimental data $I_{\exp }(\varphi)$. (b) shows the translational order $c_{G}(R)$. Here only one data set is plotted as the results overlap on the linear scale; the three data sets are replotted on a double logarithmic scale in the inset of (b), where the dashed line is a guide to the eye highlighting an algebraic decay. (c) shows the orientational order $g_{6}(R)$. Note that for clarity, the points $c_{G}(0)=1$ and $g_{6}(0)=1$ lie outside the scales used here. The colored solid lines in these plots are fits as described in the text.

fractured lattice scenario for the dislocation-free quasiordered phase, the regions of dimension $R_{d} \approx 1 \mu \mathrm{m}$ (this is independent of our RMC ensemble size $N$ ) effect a finite loss-of-neutron-correlation volume [16], giving a nondivergent Bragg peak of $\mathrm{FWHM} \approx 1^{\circ} \gg$ instrument resolution, in sharp contrast to the diverging Bragg peaks predicted for the $\mathrm{BrG}$ [9]. The elastic $\mathrm{BrG}$ theory may fail due to nonlocal effects that preserve longrange orientational order, e.g., favored nearest-neighbor directions originating from underlying anisotropies of the superconductor. At higher fields or stronger disorder strengths we envisage dislocations most likely form at boundaries between the fractured regions of the quasi- ordered phase observed, resulting in a multidomain vortex glass [13].

In summary, we have demonstrated a RMC refinement to examine disordered crystalline samples using angleresolved scattering data. In many applications the form factor is well defined. For FLs this is not the case, yet our technique generates dislocation-free FL ensembles that all accurately reproduce the Bragg peak shape, and all yield similar correlation function forms. At small scales our data strongly indicate a random manifold regime of collectively moving FLs. At large scales there is some evidence for a Bragg glass phase; however, "fracturing" of the FL ensemble is also observed.

We are grateful for helpful discussions with R.K. McGreevy, and acknowledge financial support from the Engineering and Physical Sciences Research Council of the U.K., H. Keller (University of Zürich), DanScatt, the ILL, the University of Birmingham, and from the European Commission under the 6th Framework Programme through the Key Action: Strengthening the European Research Area, Research Infrastructures, Contract No. RII3-CT2003-505925.

[1] For a review, see R. K. McGreevy, J. Phys. Condens. Matter 13, R877 (2001).

[2] A. L. Goodwin et al., Phys. Rev. Lett. 96, 047209 (2006).

[3] H. W. Sheng et al., Nature (London) 439, 419 (2006).

[4] H.E. Fischer, A. C. Barnes, and P. S. Salmon, Rep. Prog. Phys. 69, 233 (2006).

[5] A. A. Abrikosov, Sov. Phys. JETP 5, 1174 (1957).

[6] A. I. Larkin, Zh. Eksp. Teor. Fiz. 58, 1466 (1970) [Sov. Phys. JETP 31, 784 (1970)].

[7] M. V. Feigel'man et al., Phys. Rev. Lett. 63, 2303 (1989).

[8] T. Nattermann, Phys. Rev. Lett. 64, 2454 (1990).

[9] T. Giamarchi and P. Le Doussal, Phys. Rev. B 52, 1242 (1995).

[10] J. Kierfeld, T. Nattermann, and T. Hwa, Phys. Rev. B 55, 626 (1997)

[11] M. P. A. Fisher, Phys. Rev. Lett. 62, 1415 (1989).

[12] E. M. Chudnovsky, Phys. Rev. B 40, 11355 (1989).

[13] S.S. Banerjee et al., Physica (Amsterdam) 355C, 39 (2001); G. I. Menon, Phys. Rev. B 65, 104527 (2002).

[14] T. Klein et al., Nature (London) 413, 404 (2001).

[15] N. Ye. Alekseyevskiy, V.I. Nizhankovskiy, and K.-H. Berthel, Fiz. Met. Metalloved. 37, 63 (1974).

[16] R. Gähler et al., Phys. Rev. A 58, 280 (1998).

[17] E. M. Chudnovsky and R. Dickman, Phys. Rev. B 57, 2724 (1998).

[18] J. Schelten, H. Ullmaier, and W. Schmatz, Phys. Status Solidi B 48, 619 (1971); R. Kahn and G. Parette, Solid State Commun. 13, 1839 (1973).

[19] S. Bogner, T. Emig, and T. Nattermann, Phys. Rev. B 63, 174501 (2001).

[20] M. Kardar, J. Appl. Phys. 61, 3601 (1987).

[21] T. Halpin-Healy, Phys. Rev. A 42, 711 (1990).

[22] P. Kim et al., Phys. Rev. B 60, R12 589 (1999). 